\title{
Guidelines, Algorithms, Critical Pathways, Templates, and Evidence-based Medicine
}

\author{
Marvin L. Birnbaum, MD, PhD
}

The buman understanding is naturally right, and bas witbin itself a strength sufficient to arrive at the knowledge of truth, and to distinguish it from error.

Burlamaqui, Prinicples of Natural Law

There are evolving in medicine a host of guidelines, algorithms, templates, and critical pathways. Each is a significant part of the Guidelines for Evaluation and Research in the Utstein Style, the Executive Summary of which was published in the last issue, ${ }^{1}$ and the complete work will be published as a Supplement to Volume 15 in 2000. Another templatelike document is the lead article in this issue. ${ }^{2}$ The development of such patterns for the delivery or analysis of medical care is hailed by some who believe these documents provide organization out of chaos. Others object to the structure provided as attempts to "cookbook" medical care. Others point out that the codification of such processes threatens the important role played by "experience" and "logic"; still others insist that such devices establish "standards" and that deviations from these standards present an increased risk for legal actions. Thus, confusion reigns relative to the meaning and utility of such documents. And, to confuse matters further, there now is a drive by some to evolve what they call, "evidence-based medicine". Are these all the same?

It is assumed by many that all of the above documents have been and will be based upon "scientific evidence". Evidence consists of data upon which a judgment or conclusion may be based. Evidence must be "valid" 3,4 Evidence-based medicine, therefore, supposedly is based upon facts. A fact is a thing that is known to bave occurred, to exist, or to be true; it is reality. ${ }^{5}$ It seems clear that hypotheses proven by quantitative studies that have established a cause:effect relationship with bigh probability of being a fact $(p$ $\geq 0.95$; error $\leq 0.05$ ), constitute evidence and generally have high internal validity. But, can we use information defined by qualitative techniques as evidence? Surely these data have high external validity and gain internal validity with repeated documentation.

Thus, it is cogent to examine the process by which some of these instruments have been devised. My first experience with the use of guidelines and algorithms came in 1974 with the development and publication of the Standards for Cardiopulmonary Resuscitation and Emergency Cardiac Care. ${ }^{6}$ It is curious that this document contained algorithms (a process or set of rules used for problem-solving ${ }^{7}$ ) that became accepted as the "rules" that established a structure for the practice of resuscitology. They became a "standard" of practice: a "standard" is defined as an object or quality or measure serving as a basis or example or principle to which others conform or should conform or by which the accuracy or quality of others is judged. ${ }^{8}$ The importance of having standards is discussed in detail in the Continuing Education lesson in this issue. ${ }^{9}$ Were these standards an early example of evidence-based medicine?

It is important to recognize that the process used for their development established the pattern used subsequently to evolve other medical standards. These standards resulted from a comprehensive review of the available literature by a panel of experts: where facts had been established, they were included; where facts did not exist or were disputed, the "facts" were tempered by the practice experiences of many, and the standards ultimately were agreed upon by consensus. What actually was published was a combination of fact and opinion. In essence, the "standards" were the amalgamation of quantitative and qualitative research!

It also is curious that in its subsequent publications, the AHA changed the title from "Standards" to "Guidelines". ${ }^{10} \mathrm{~A}$ guideline is defined as $a$ principle or criterion guiding or directing action, or a statement of policy by a person or group baving the authority. ${ }^{11}$ 'Thus, the AHA recognized that in fact, it had established standards for which it did not wish to be responsible legally. However, the use of these algorithms did not change with the name change.

These algorithms essentially constitute the first examples of critical pathways in our field. They represent what was considered by experts in our field as the "best" way to practice in a given set of circumstances given the current state of knowledge and experience. They served as excellent models upon which to base teaching. Ultimately, as new knowledge, experience, and evidence accumulated relative to these critical pathways, deviations from the pathways occurred. Often such deviations became integrated into a new set of revised standards. Thus, we learned that standards and critical pathways are not chiseled in stone, but must be evaluated continually and modified as new information accumulates. Critical pathways really represent a tool for the ongoing development of "standards" and definition and redefinition of facts. And this is the intent of the forthcoming "Guidelines".

Lastly, it is worthwhile to examine templates and their purpose. Templates are patterns that provide structure. ${ }^{12}$ Such was the intent in the development of the initial Utstein Templates. ${ }^{13-17}$ It was clear that the reporting of data derived from the study of victims of cardiac arrest was unpatterned and 
hence, different studies could not be analyzed in a similar fashion. In order to establish a pattern for data collection and analysis that would lend itself to analysis of the "big picture", it was necessary to establish a minimum data set to be used universally in reporting studies of cardiac arrest. The Template for reporting data from cardiac arrest was developed with the prodding of Tore Laerdal, by a group of recognized experts at the Utstein Abbey in Stavanger, Norway. Subsequently, this methodology of establishing patterns for reporting data that previously had been unstructured and defuse has been given the title of the "Utstein Style".

The Templates presented in this issue for the reporting of data acquired from victims of major trauma establishes the basic structure necessary to make sense out of the plethora of data being collected. The same will hold for the development of research techniques and reporting of data regarding medical responses to disasters, and hopefully, for all disaster responses. Like standards, templates require continuous re-examination, and the data gathering instruments must be developed with flexibility in mind. But, once data collection begins in the formats suggested, it is not fruitful to change the structure. Therefore, it is essential that as the Templates are developed and implemented, great care be taken in selecting the structure. The development of computer databases to allow the analysis of large volumes of data is the next step. We all look forward to the initial development of these databases. In the disciplines of Emergency and Disaster Medicine, this is an essential step to the development of our specific science.

I urge your critical analysis of these guidelines, algorithms, critical pathways, and templates. They constitute evidence-based medicine and will form the standards of our practice. They should be gratefully accepted, and evaluated, and commented upon (input welcomed on the PDM website (http://pdm.medicine.wisc.edu)), and will evolve. Guidelines, algorithms, critical pathways, and templates are not the same. Each has a specific role. But, it only is through the use and development of such tools that our science will gain the respect it deserves.

Facts when combined with ideas, constitute the greatest force in the world. They are greater than armaments, greater than finance, greater than science, business, and law because thay are the common denominator of all of them.

Carl W. Ackerman, 1931

\section{References}

1. Task Force on Quality Control of Disaster Management, KO Sundnes, Chair: Health disaster management: Guidelines for evaluation and research in the Utstein Style. Prebospital Disaster Med 1999;14:43-52.

2. International Trauma, Anaesthesia, and Critical Care Society: Recommendation for uniform reporting of data following major trauma - The Utstein Style: An intitiative. Prehospital Disaster Med 1999;14:13-40.

3. Morris W (ed): American Heritage Dictionary of the English Language, Houghton Mifflin Co. Geneva, IL, 1980. p 455.

4. Morris W (ed): American Heritage Dictionary of the English Language, Houghton Mifflin Co. Geneva, IL, 1980. p 455.

5. Thompson D (ed): The Concise Oxford Dictionary of Current English. 9th ed., Clarendon Press: Oxford. 1995. p 467.

6. Ibid, $\mathrm{p} 482$.

7. American Heart Association: Standards for cardiopulmonary resuscitation and emergency cardiac care. JAMA 1974;227:833-868.

8. Thompson D (ed): The Concise Oxford Dictionary of Current English. 9th ed., Clarendon Press: Oxford. 1995. p 32.

9. Ibid., 1357.

10. Cuny FC: Principles of disaster management: Lesson 5: Program supervision, monitoring, and control. Prehospital Disaster Med 1999:14:198-210.

11. Emergency Cardiac Care Committee and Subcommittees, American Heart Association: Guidelines for cardiopulmonary resuscitation and emergency cardiac care. JAMA 1992;268:2171-2302.

12. Morris W (ed): American Heritage Dictionary of the English Language, Houghton Muffin Co. Geneva, IL, 1980, p 585.

13. Ibid, 1325.

14. "Special report: Recommended guidelines for uniform reporting of data from out-of-hospital cardiac arrest: The Utstein Style. A statement for health professionals from a task force of the American Heart Association, the European Resuscitation Council, the Heart and Stroke Foundation of Canada, and the Australian Resuscitation Council. Circulation 1991;84:960-975.
15. Recommended guidelines for uniform reporting of data from out-of-hospital cardiac arrest: The Utstein Style. A statement for health professionals from a task force of the American Heart Association, the European Resuscitation Council, the Heart and Stroke Foundation of Canada, and the Australian Resuscitation Council. Resuscitation 1991;22:1-26.

16. Task Force of the American Heart Association, the European Resuscitation Council, the Heart and Stroke Foundation of Canada, and the Australian Resuscitation Council: Recommended guidelines for the reporting of out-of-hospital cardiac arrest: The Utstein Style. Ann Emerg Med 1991;20:861-874.

17. Task force of representatives from the European Resuscitation Council, American Heart Association, Heart and Stroke Foundation of Canada, and the Australian Resuscitation Council: Recommended Guidelines for uniform reporting of data from out-of-hospital cardiac arrest (new abridged version): The "Utstein-Style," Br Heart J 1992;67:325-332.

Editor's Note: PDM welcomes the National Library of Medicine to indexing the science in PDM. It represents an important addition that will make PDM more accessible to researchers. PDM now is indexed by the National Library of Medicine (Medline), the Cumulative Index for Nursing and Allied Health Life Sciences (CINAHLS), and Healthstar. In addition, comprehensive searches of PDM are available via the PDM website (http://pdm.medicine.wisc.edu). All of these indexing services provide access to the important information published in PDM, and each indexes back to our initial issues. Authors, please take note. 